Boletín de la Sociedad Zoológica del Uruguay, 2020

Vol. 29 (2): 99-105

ISSN 2393-6940

https://journal.szu.org.uy

\title{
APORTES AL ESTUDIO DE Trichocera (Saltrichocera) maculipennis Meigen, 1818 (DIPTERA: TRICHOCERIDAE) EN LA ISLA REY JORGE
}

\author{
Mónica Remedios-De León ${ }^{1}$; Martín Santana ${ }^{1}$; Damián Hagopián¹; Gabriela Bentancur ${ }^{1}$ \& \\ Enrique Morelli ${ }^{1}$
}

1. Sección Entomología, Facultad de Ciencias. Iguá 4225. Montevideo, Uruguay.

Autor para correspondencia: mremedios@fcien.edu.uy

\section{RESUMEN}

Las invasiones biológicas se encuentran entre las amenazas más importantes para la biodiversidad a nivel mundial. En general la introducción de nuevas especies en regiones geográficas fuera de su lugar de origen se ha producido por razones económicas, científicas y sociales teniendo un impacto, muchas veces con consecuencias imprevistas. La Antártida es uno de los lugares más remotos de la Tierra y uno de los hábitats más prístinos. El aislamiento del continente Antártico llevaría a pensar que cuenta con pocas especies invasoras, sin embargo, desde la llegada de los humanos a las islas subantárticas, se ha registrado un aumento de especies no autóctonas. La mayoría de las especies introducidas en el territorio Antártico no han podido sobrevivir a las condiciones climáticas, pero hay otras que si lo han hecho como es el caso del díptero Trichocera (Saltrichocera) maculipennis Meigen, 1818. Dicha especie se registro por primera vez en 2006 en la Base Científica Antártica Artigas y continúa siendo reportada en varias Bases científicas antárticas. Este trabajo presenta datos sobre la abundancia de T. maculipennis en sitios de la Base Científica Antártica Artigas de la Isla Rey Jorge mediante el empleo y la efectividad de trampas alternativas utilizadas en el muestreo de esta especie. Se discuten los datos obtenidos a la luz de planes de mitigación hacia programa de control y erradicación más efectivos de esta especie en instalaciones de las Bases Científicas de la Isla Rey Jorge y evaluar la efectividad de trampas de pegamento utilizadas para el muestreo de la especie.

Palabras claves: Díptero. Especie introducida. Antártida.

\section{ABSTRACT}

Contributions to the study of Trichocera (Saltrichocera) maculipennis Meigen, 1818 (Diptera: Trichoceridae) on the Island Rey Jorge. Biological invasions are among the most important threats to biodiversity worldwide. In general, the introduction of new species in geographical regions outside their place origin has occurred for economic, scientific and social reasons, having an impact, often with unforeseen consequences. Antarctica is one of the most remote places on Earth and one of the most pristine habitats. The isolation of the Antarctic continent would lead to think that it has few invasive species, however, since the arrival of humans to the subantarctic islands, there has been an increase in non-native species. Most of the species introduced into the Antarctic territory have not been able to survive the climatic conditions, but there are others that have, such as the dipteran Trichocera (Saltrichocera) maculipennis Meigen, 1818. This species was recorded for the first time in 2006 in the Artigas Antarctic Scientific Base and continues to be reported in several Antarctic scientific bases. This work presents data on the abundance of $T$. maculipennis at sites of the Artigas Antarctic Scientific Base on King George Island through the use and effectiveness of alternative traps used in the sampling of this species. The data obtained in the light of mitigation plans towards a more effective control and eradication program of this species in facilities of the Scientific Bases of Isla Rey Jorge are discussed and the effectiveness of the glue traps used for the sampling of the species is evaluated.

Key words: Diptera. Invasive species. Antarctica.

\section{INTRODUCCIÓN}

Los ecosistemas terrestres en el continente Antártico se limitan a áreas libres de hielo, representando el $0.2 \%$ de su superficie (Convey, 2007; Hughes \& Pertierra, 2016; Burton-Johnson et al., 2016). Las bajas temperaturas, los vientos fuertes, la corta temporada de vegetación y las áreas limitadas sin hielo hacen que la Antártida sea inhóspita para la mayoría de los organismos terrestres. La biodiversidad de flora está limitada a algas, líquenes y musgos y la fauna principalmente a microinvertebrados y dos especies de macroartrópodos, Belgica antarctica Jacobs, 1900 y Parachlus steinenii Gercke, 1889 (Smith, 1984; Frenot et al., 2005; Hughes \& Pertierra, 2016). La aparición 
de especies no nativas, especialmente aquellas que se puedan establecer con éxito en el área, debe considerarse como una amenaza para los ecosistemas terrestres antárticos y su biodiversidad (Comité para la Protección del Medio Ambiente, 2016). Las invasiones biológicas se encuentran entre las amenazas más importantes para la biodiversidad en la tierra (McKinney \& Lockwood, 1999; McNeely et al., 2001). Este fenómeno representa un gran riesgo a la supervivencia de las especies nativas y son responsables de los mayores cambios en la estructura y funcionamiento de los ecosistemas (Mack et al., 2000; Scott \& Kirkpatrick, 2008). A nivel mundial, las introducciones de especies invasoras se han producido por razones económicas, científicas, sociales y con consecuencias imprevistas (Mack et al., 2000). Estas especies introducidas, naturalmente no poseen depredadores en su nuevo ambiente, por lo cual, carecen de controladores naturales (McNeely et al., 2001).

El aislamiento del continente Antártida llevaría a pensar que cuenta con pocas especies invasoras, en comparación con otras regiones de la Tierra (Frenot et al., 2005; Hughes \& Coney, 2010), sin embargo, desde la llegada de los humanos a las islas subantárticas a fines del siglo XVIII y principios del XIX, la cantidad de especies introducidas han aumentado (Frenot et al., 2005; Convey \& Lebouvier, 2009). Las zonas Antárticas en las que se han registrado mayor influencia de especies invasoras, son aquellas que se encuentran más cercanas al continente Sudamericano y con un mayor impacto antropogénico (Frenot et al., 2005; Whinam et al., 2005; Hughes et al., 2011; Convey et al., 2012; Hughes \& Convey, 2014). Debido a estas preocupaciones, se aprobó el Protocolo del Tratado Antártico sobre Protección Ambiental, el cual prohíbe la introducción intencional de especies no autóctonas en la Antártida, excepto con un permiso expedido y que estipula su remoción o eliminación antes de la expiración de dicho permiso (Potocka \& Krzeminska, 2018). Las especies introducidas se han convertido en una gran amenaza para las comunidades antárticas (Hughes et al., 2015) la mayoría de ellas no han podido sobrevivir a las condiciones climáticas, mientras otras si lo han hecho (Greenslade \& Convey, 2017; Olech \& Chwedorzewska, 2011) como es el caso del díptero Trichocera (S) maculipennis Meigen 1818. Trichocera macullipennis se registró por primera vez en 2006 en la Base Científica Antártica Artigas (Hughes et al. 2015). Esta especie continúa siendo reportada en varias bases científicas antárticas, por lo cual preocupa su estrategia de dispersión. Se ha observado asociada fundamentalmente a asentamientos humanos donde pueden reproducirse en condiciones más favorables (Hughes et al., 2005). Potocka \& Krzeminska (2018) observan esta especie en la Estación Arctowski de Polonia en Admiralty Bay, Isla Rey Jorge en 2017.
Trichocera maculipennis se distribuye principalmente en las regiones Holárticas tolerando tanto climas cálido como fríos (Petrasiunas \& Paramonov, 2014) y en el hemisferio sur se conoce para las islas Kerguelen (Séguy, 1940; Séguy, 1953) y Rey Jorge (Volonterio et al., 2013).

La larva de T. maculipennis es similar a la de todas las especies del género (Rhynehart, 1925; Brindle, 1962), presentando una cápsula cefálica oscura, esclerotizada y parcialmente retráctil. Los primeros cuatro segmentos detrás de la cabeza se dividen secundariamente en dos y los siguientes seis en tres partes. El último, tiene cuatro lóbulos carnosos, dos dorsales y dos ventrales y entre ellos, se sitúa un par de espiráculos (Potocka \& Krzeminska, 2018). En general las larvas de tricocéridos son terrestres, mientras que las de $T$. maculipennis pueden vivir en sustrato semilíquido o líquido ya que pueden elevar el extremo anal con sus espiráculos por encima de la superficie para poder respirar (Karandikar, 1931). Todas las etapas en el ciclo de vida de T. maculipennis son vulnerables a la desecación, al congelamiento o al calentamiento, temperaturas inferiores $\mathrm{a}-10^{\circ} \mathrm{C}$ y superiores a $32^{\circ}$ $C$ pueden llegar a matar a los adultos y las larvas (Dahl, 1969). Se adaptaron a los ambientes urbanizados, ambientes oscuros, como sótanos y almacenes (Grimshaw, 1906 ), pero también en cuevas naturales donde las condiciones de temperatura son estables (Petrasiunas \& Weber, 2013). Estos hábitats naturales proporcionan ricas fuentes de alimento para las larvas como guano, abono y desechos de aguas residuales (Potocka \& Krzeminska, 2018). Según Karandikar (1931), la duración del ciclo de vida depende en gran medida de las condiciones ambientales y puede ir desde 40 días a un año. Hasta ahora se desconocen los medios de propagación (Potocka \& Krzeminska, 2018), la especie puede volar a temperaturas del entorno de los $0^{\circ} \mathrm{C}$, por lo que el vuelo parece una posibilidad, pero los Trichoceridae son malos voladores y el vuelo no puede tener lugar en condiciones de viento (Dahl, 1965). Las especies invasoras son difíciles de erradicar especialmente en ecosistemas tan frágiles como la Antártida terrestre, donde se deben seguir normas muy estrictas en el uso de insecticidas y aplicar lo antes posible aquellas medidas adoptadas conjuntamente por todas las partes (Hughes et al., 2005). Los primeros pasos para establecer un plan de acción para T. maculipennis en la isla Rey Jorge fueron tomados conjuntamente por Uruguay, Corea, Chile y Reino Unido (ATCM XL - WP 26, 2017). Todos los países presentes en la Isla Rey Jorge son conscientes del problema de la presencia de esta especie no-nativa y en conjunto se está realizando un monitoreo sistemático (Potocka \& Krzeminska, 2018). El control o la erradicación solo pueden tener éxito mediante la aplicación de medidas de 
bioseguridad estrictas y eficaces por parte de todos los operadores nacionales que utilizan la isla Rey Jorge y las islas Shetland del Sur. Este trabajo tiene como objetivo actualizar el estado de la presencia de T. maculipennis en diferentes Bases Científicas de la Isla Rey Jorge y evaluar la efectividad de trampas de pegamento utilizadas para el muestreo de esta especie.

\section{MATERIALES Y MÉTODOS}

El área de estudio fue la Base Científica Antártica Artigas (62011'S, 58 $\left.51^{\prime} \mathrm{W}\right)$. Los muestreos se realizaron durante los años 2018-2019. Se emplearon dos técnicas de recolección: trampas pitfall (de caída) durante los muestreos estivales en los meses de enero y febrero y trampas de pegamento que estuvieron activas durante todo el año en la Base, recambiando la plancha de pegamento una vez al mes. Las trampas pitfall se consideran más adecuadas para el monitoreo exterior. Las trampas de pegamento se adecuan a la captura dentro de las instalaciones de la Base ya que las condiciones climáticas disminuyen significativamente su efectividad. Se colocaron 13 trampas pitfall en un radio de $1000 \mathrm{~m}$ en el entorno de la Base, que fueron retiradas a los 5 días. Se utilizó alcohol $70 \%$ como liquido conservante. Se colocaron 20 trampas de pegamento dentro de las instalaciones de la Base (hangar, tanque de agua, almacén, área de servicio, caseta del jefe de base, caseta radio comunicaciones, tanque radio AINA, tratamiento de residuos, caseta comedor, oficina del jefe de base, depósito de agua sector radio, cámara séptica AINA, caseta alojamiento de la dotación, depósito AINA, sala de equipo de aire, contenedor de la playa, lavadero y caseta laboratorio). La identificación sistemática se basó en la estructura alar según la metodología de Dahl (1966). La separación de machos y hembras se realizó examinando la genitália externa de los adultos. Se separó un lote de ejemplares que fueron depositados de Entomología de la Facultad de Ciencias.

\section{RESULTADOS}

Se recolectaron un total de 7231 adultos, 385 en trampas pitfall y 6846 en trampas de pegamento en todo el período de estudio. Los datos de colecta en ambos años muestran que la abundancia de ejemplares recolectados mediante trampas de pegamento, $\mathrm{N}=5044$ (2018) y $\mathrm{N}=$ (1802) (2019), fueron significativamente mayores que aquellos recolectados con trampas pitfall $\mathrm{N}=102$ (2018) y $\mathrm{N}=172$ (2019). La Tabla 1 presenta la relación de abundancia en los 20 sitios de muestreo para los años 2018 y 2019 con trampas de pegamento. La trampa con mayor abundancia fue la ubicada en la cámara séptica del AINA (sitio 13).

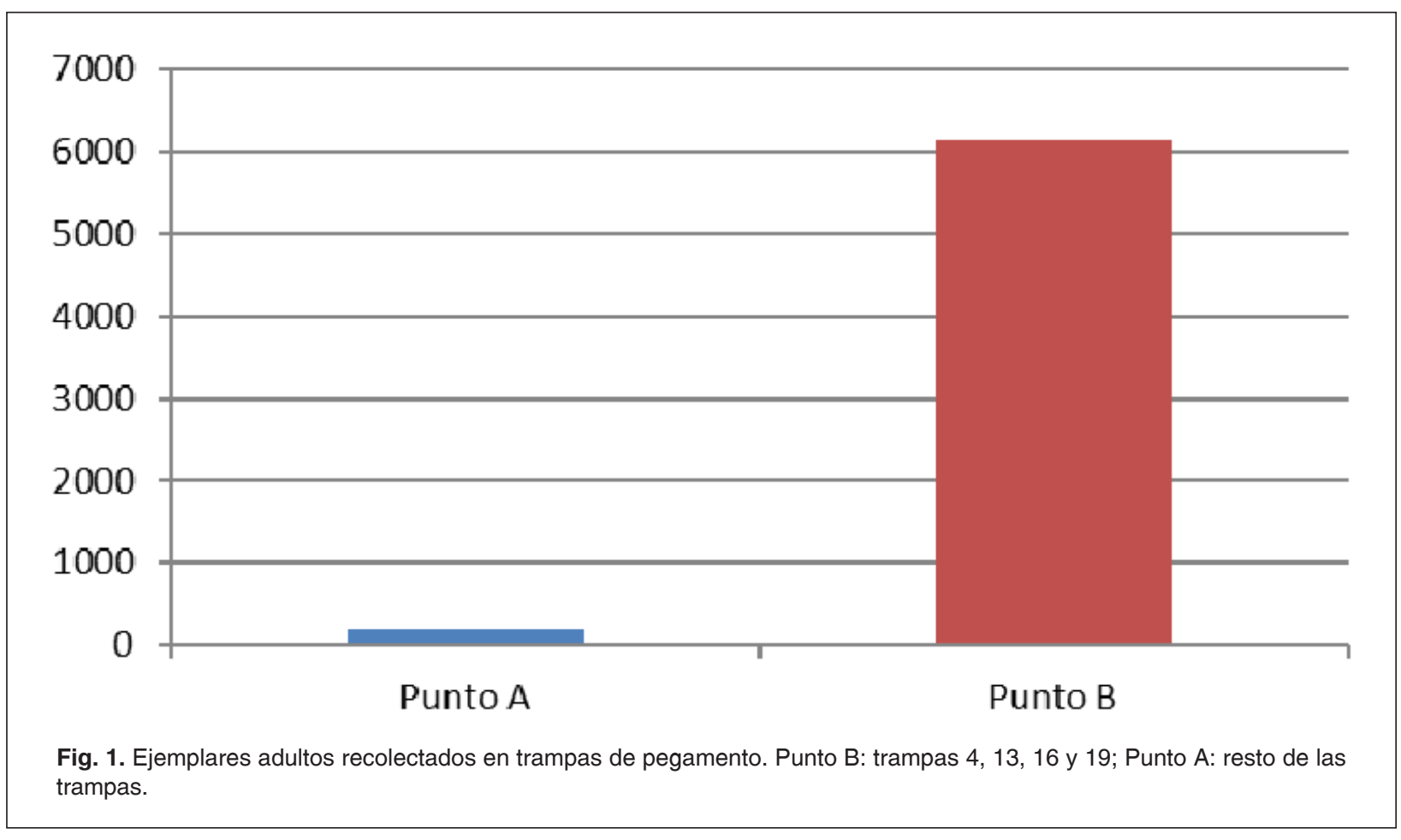

Bol. Soc. Zool. Uruguay (2ª época). 2020. Vol. 29 (2): 99-105 ISSN 2393-6940 
Tabla 1. Abundancia de ejempalres recolectados en 20 sitios de colecta en las instalaciones de la Base Cientifica Antártica Artigas utilizando trampas de pegamento.

\begin{tabular}{lrrr}
\multicolumn{1}{c}{ Sitio de colecta } & N (2018) & N (2019) & Total \\
\hline Sitio 1. Hangar & 1 & 1 & 2 \\
Sitio 2. Tanque de agua & 22 & 13 & 35 \\
Sitio 3. Almacén área de servicio & 0 & 4 & 4 \\
Sitio 4. Caseta jefe de Base & 31 & 156 & 187 \\
Sitio 5. Cocina & 0 & 26 & 26 \\
Sitio 6. Caseta radio comunicaciones & 2 & 1 & 3 \\
Sitio 7. Tanque agua AINA & 14 & 15 & 29 \\
Sitio 8. Tratamiento de residuos & 1 & 1 & 2 \\
Sitio 9. Caseta comedor & 6 & 11 & 17 \\
Sitio 10. Almacén & 0 & 0 & 0 \\
Sitio 11. Oficina del jefe de Base & 3 & 12 & 15 \\
Sitio 12. Depósito de agua sector radio & 1 & 0 & 1 \\
Sitio 13. Cámara séptica AINA & 4816 & 1029 & 5845 \\
Sitio 14. Caseta alojamiento dotación & 1 & 0 & 1 \\
Sitio 15. AlNA depósito & 0 & 0 & 0 \\
Sitio 16. Caseta AlNA & 32 & 29 & 51 \\
Sitio 17. Sala de aire & 0 & 7 & 7 \\
Sitio 18. Contenedor de la playa & 5 & 4 & 9 \\
Sitio 19. Lavadero & 6 & 65 & 71 \\
Sitio 20. Caseta laboratorio & 0 & 46 & 46 \\
\hline
\end{tabular}

La figura 1 muestra el agrupamiento de la abundancia observada en los 20 sitios de muestreos. Punto A muestras con abundancias entre 0 y 50 ejemplares (puntos 1, 2, 3, 5, 6, 7, 8, 9, 10, 11, 12, 14, $15,17,18,20)$ y Grupo B con más de 50 ejemplares (punto $4 ; 13 ; 16$ y 19). La cámara séptica AINA (punto 13) contribuye a la mayor abundancia del Grupo B.

La Fig. 2 muestra la relación de los sexos en cada agrupamiento. Es notorio que la relación entre los sexos en el Punto B es significativamente diferente en machos y hembras. Este dato es coincidente con la relación entre los sexos observada en la cámara séptica AINA (sitio 13) (Fig. 3), sitio de mayor contribución en las abundancias del Punto B.

\section{DISCUSIÓN}

Nuestros resultados evidencian que las muestras realizadas en la cámara séptica AINA (sitio 13) presentan el mayor número de ejemplares recolectados, esto indica que allí se encuentran las mejores condiciones ambientales para postura, desarrollo y emergencia de adultos de $T$. maculipennis. Entre los sitios aquí analizados en instalaciones de la Base Científica Antártica Artigas de la Isla Rey Jorge. La caseta del jefe y caseta del AINA (sitios 4 y 16) con abundancias de más de 50 ejemplares pueden ser preferidos por sus características de área de refugio para el insecto adulto y su proximidad al punto 13 considerado como punto de emergencia y dispersión. Los lugares con abundancias menores a 50 serían áreas que podrían oficiar como refugios transitorios del adulto. Los puntos con abundancias entre 0 y 5 ejemplares podrían ser menos atractivos por ser ambientes abiertos, ventilados y con tránsito permanente de vehículos y personal, representando áreas poco atractivas para una colonización estabilizada. Este trabajo utilizo por primera vez la metodología de trampas de pegamento en la Base, estas trampas de pegamento adquieren una doble función permitiendo por un lado realizar un monitoreo continuo de $T$. maculipennis y a su vez colaborar activamente en el control poblacional de esta especie. La notoria disminución de capturas en la cámara séptica del AINA reportada en muestras del 2019 (ver Tabla 1) puede haber sido influida por la colocación en 

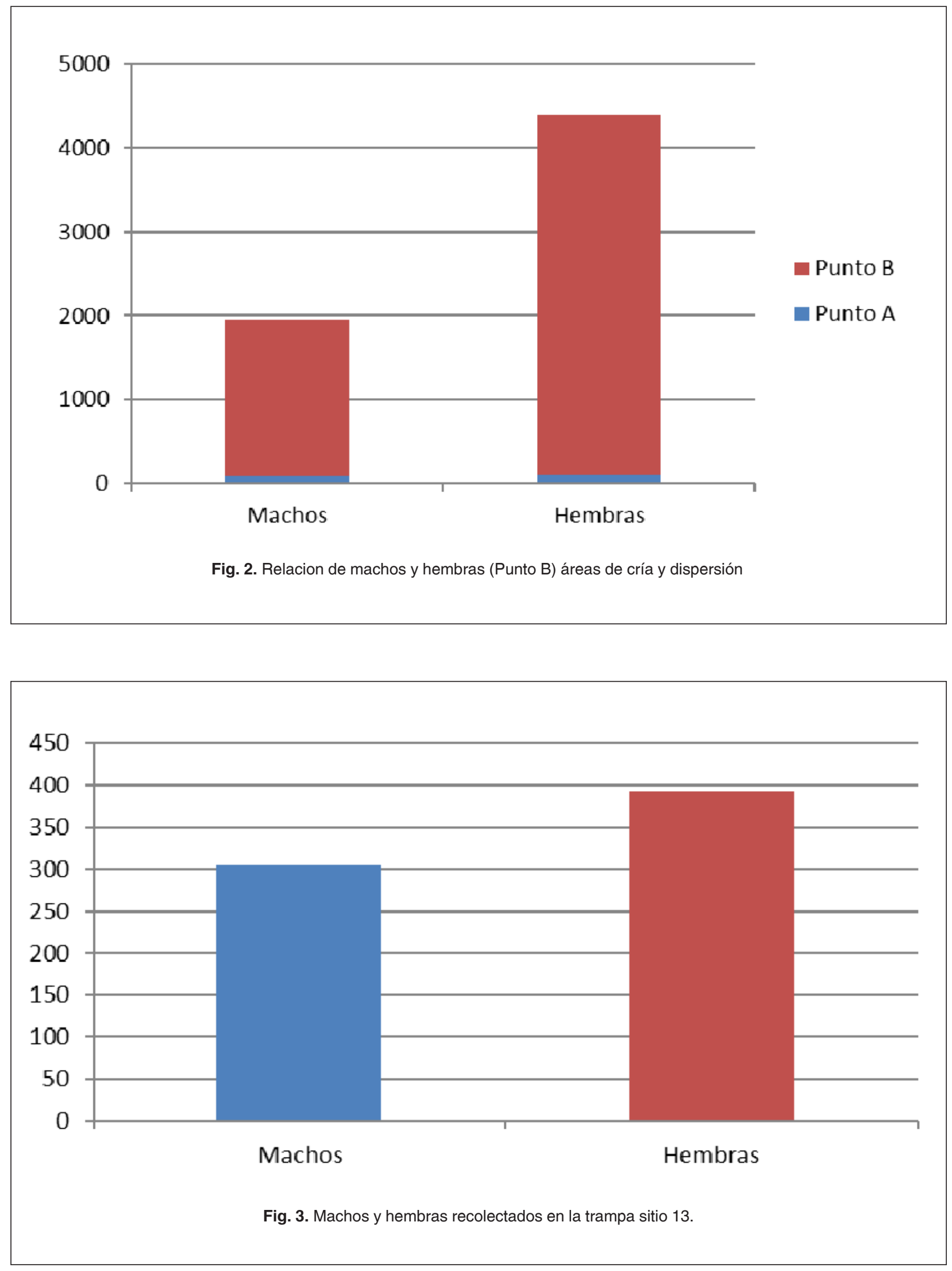
conjunto de trampas de luz ultravioleta y de pegamento en el interior de la cámara.

\section{CONCLUSIONES}

Este artículo presenta nuevos datos de registro de recolección de la especie Trichocera maculipennis en la Base Científica Antártica Artigas para los años 2018 y 2019. Estudios realizados en la Base de Polonia también coinciden en la identificación de áreas de emergencia y dispersión en instalación de cámara séptica (Potocka \& Krzeminska, 2018). Estos lugares reúnen condiciones de temperatura y resguardo para los adultos, presencia de agua y materia orgánica necesarios para postura y desarrollo de las larvas. En los sucesivos años de monitoreo se registró la presencia de la especie tanto dentro como fuera de las instalaciones de la Base. Las trampas de pegamento mostraron ser de gran eficiencia para la captura de ejemplares adultos de T. maculipennis, por lo tanto, es importante su consideración a la hora de implementar un plan de monitoreo y control. Hay que tener presente que las especies invasoras pueden llegar a tener un papel ecológico muy importante en el ecosistema que ocupan. Para el control de las especies no-nativas es necesario un plan de erradicación estricto y desarrollado en base a datos científicos actualizados por los operadores nacionales que utilizan la isla Rey Jorge. Si T. maculipennis logra colonizar el entorno natural los protocolos de erradicación serán inútiles. Un protocolo de mitigación y eventual erradicación definitiva de la especie debería contemplar al menos las siguientes recomendaciones: -Monitoreo regular de sitios de localización de la especie, números de ejemplares recolectados en cada uno y circuitos de dispersión.

- Identificación de los períodos con mayor abundancia de ejemplares, así como detectar los sitios de cría de larvas. Esto permitirá establecer una reingeniería de sitios de cría como cámaras sépticas o tubos de ventilación e implementar prácticas manuales de limpieza en los lugares de alto riesgo de cultivo de esta especie.

- Teniendo en cuenta los resultados aquí presentados se puede minimizar el número de trampas utilizadas para un monitoreo control al identificar los lugares de cría y dispersión.

\section{AGRADECIMIENTOS}

Este trabajo se desarrolla en el marco de un proyecto del Instituto Antártico Uruguayo. Se agradece al personal de la Base Científica Antártica Artigas presente en las campañas 2018-2019 por el apoyo logístico y a los revisores anónimos por los aportes que enriquecieron este trabajo.

\section{BIBLIOGRAFÍA}

Brindle A. 1962. Taxonomic notes on the larvae of British Diptera. II. Trichoceridae and Anisopodidae. The Entomologist, 95:285-288.

Burton-Johnson A., Black M., Fretwell P.T. \& J. KaluzaGilbert. 2016. An automated methodology for differentiating rock from snow, clouds and sea in Antarctica from Landsat imagery: a new rock outcrop map and area estimation for the entire Antarctic continent. Cryosphere 10:1665-1677 DOI 10.5194/tc-10-1665-2016.

Chwedorzewska K., Korczak-Abshire M., Olech M., LityD ska-Zaj c M. \& A. Augustyniukkram. 2013. Alien invertebrates transported accidentally to the Polish Antarctic Station in cargo and on fresh foods. Polish Polar Research, 34:55-66 DOI 10.2478/popore-2013-0005.

Committee for Environmental Protection. 2016. CEP non-native species manual. Secretariat of the Antarctic Treaty. Available at https://www.ats.aq/ documents/ recatt/ att608_e.pdf (accessed on 4 April 2016).

Convey P. 2007. Antarctic ecosystems. In: Levin SA, ed. Encyclopedia of biodiversity. 2nd (online) edition. San Diego: Elsevier. Convey P, Block W. 1996. Antarctic Diptera: ecology, physiology and distribution. European Journal of Entomology, 93:1-13.

Convey P. \& M. Lebouvier. 2009. Environmental change and human impacts on terrestrial ecosystems of the sub-Antarctic islands between their discovery and the mid-Twentieth Century. Papers and Proceedings of the Royal Society of Tasmania, 143: 33-44.

Convey P., Barnes D.K., Griffiths H., Grant S., Linse K. \& D. Thomas. 2012. Biogeography and regional classifications of Antarctica. Chapter 16 in A.D. Rogers, N.M. Johnston, E. Murphy, and A. Clarke, editors. Antarctica: an extreme environment in a changing world. Blackwell, Oxford, UK.

Convey P., Coulson S., Worland M.R. \& A. Sjöblom. 2018. The importance of understanding annual and shorter-term temperature patterns and variation in the surface levels of polar soils for terrestrial biota. Polar Biology, 1-19 DOI 10.1007/ s00300-018-2299-0.

Dahl C. 1965. Studies on warming activity in Trichoceridae (Dipetra) in southern Sweden. Opuscula Entomologica, Supplementum, 27:568.

Dahl C. 1969. The influence of light, humidity and temperature on Trichoceridae (Diptera). Oikos, 20:409-430 DOI 10.2307/3543206.

Edwards F.W. 1928. Diptera Fam. Protorhyphidae, Anisopodidae, Pachyneuridae, Trichoceridae. In: Wytsman P, ed. Genera insectorum. Vol. 
190. Bruxelles: DesmetVerteneuil, 1-41.

Edwards J.A. \& D.M. Greene 1973. The survival of Falkland Islands transplants at South Georgia and Signy Island, South Orkney Islands. British Antarctic Survey Bulletin, 33- 34:33-45.

Frenot Y., Chown S.L., Whinam J., Selkirk P.M., Convey P., Skotnicki M. \& D.M. Bergstorm 2005. Biological invasions in the Antarctic: extent, impacts and implications. Biological Reviews of the Cambridge Philosophical Society, 80:45-72 DOI 10.1017/S1464793104006542.

Grimshaw P.H. 1906. On the occurrence of a new British fly Trichocera maculipennis in the fourth district. Annals of Scottish Natural History, 15:210-211.

Hughes K.A. \& P. Convey. 2014. Alien invasions in Antarctica is anyone liable? Polar Res. 33, 22103. polar.v33.22103.

Hughes K.A., Walsh S., Convey P., Richards S. \& D.M. Bergstrom. 2005. Alien fly populations established at two Antarctic research stations. Polar Biol., 28, 568-570.IAATO, 2015. Guidelines and resource (http://iaato.org/en_GB/ guidelines-and-resources. Accessed: $3 \mathrm{Nov}$ 2015)

Hughes K.A. \& P. Convey. 2010. Determining the native/ non-native status of newly discovered terrestrial and freshwater species in Antarctica-current knowledge, methodology and management action. J Environ Manag, 93:52-66.

Hughes K.A., Lee J.E., Tsujimoto M., Imura S., Bergstorm D.M., Ware C. Lebouvier M., Huiskes H.L., Gremmen J.M., Frenot Y., Bridge P.D. \& S.L. Chown. 2011. Food for thought: risk of non-native species transfer to the Antarctic region with fresh produce. Biological Conservation, 144:1682-1689 DOI 10.1016/ j.biocon.2011.03.001.

Hughes K. \& L.R. Pertierra. 2016. Evaluation of nonnative species policy development and implementation within the Antarctic Treaty area. Biological Conservation, 200:149-159 DOI 10.1016/j.biocon.2016.03.011.

Hughes K.A., Pertierra L.R., Molina-Montenegro M. \& P. Convey. 2015. Biological invasions in Antarctica: what is the current status and can we respond? Biodiversity Conservation, 24:10311055 DOI 10.1007/s10531-015-0896-6.

Karandikar K.R. 1931. The early stages and bionomics of Trichocera maculipennis (Meig.) (Diptera, Tipulidae). Transactions of the Royal Entomological Society of London, 79:249-260.

Santiago Chile. Korea, Uruguay, Chile, United Kingdom. 2017. Inter-Parties' action plan to manage the non-native flies in King George Island, South Shetland Islands. In: ATCM XL WP 26.

Mack R.N., Simberloff D., Lonsdale W.M., Evans H.,
Clout M. \& F.A. Bazzaz. 2000. Biotic invasions: causes, epidemiology, global consequences, and control. Ecol. Applic., 10: 689-710.

McKinney M.L. \& J. Lockwood. 1999. Biotic homogenization: a few winners replacing many losers in the next mass extinction. Trends Ecol. Evol., 14: 450-453.

Olech M. \& K.J. Chwedorzewska. 2011. The first appearance and establishement of an alien vascular plant in natural habitats on the forefield of retreating glacier in Antarctica. Antarctic Sciences, 23:153-154 DOI 10.1017/ S0954102010000982.

Petrasiunas A.V. \& N.M. Paramonov. 2014. New data on the distribution of winter gnats (Diptera, Trichoceridae) in Russia. Entomological Review, 94:190-199 DOI 10.1134/S0013873814020055.

Petrasiunas A.V. \& D. Weber. 2013. Winter crane flies (Insecta, Diptera, Trichoceridae) from caves of the Grand Duchy of Luxembourg. Ferrantia, 69:276-283.

Potocka M. \& E. Krzeminska. 2018. Trichocera maculipennis (Diptera) -an invasive species in Maritime Antarctica. PeerJ.DOI: 10.7717/ peerj.5408

Rhynehart J.G. 1925. The larva and pupa of Trichocera regelationis L. (Diptera, Rhyphidae) 1925. Belfast: Belfast Natural History and Philosophical Society, Session 1922-23 Plates I-III, 36-47.

Séguy E. 1940. IV Diptères. In: Jeannel R, ed. Crosière du Bougainville aux îles fran, caises. New series 14, Paris: Mémoires du Museum d'Histoire naturelle, 203-267.

Séguy E. 1953. Insectes Mallophages, Anoplures et Dipteres recueillis par M. P. Paulian aux lles Kerguelen. Mémoires de l'Institut Scientifique de Madagascar 4:553-615.

Smith R.I.L. 1984. Terrestrial plant biology of the subAntarctic and Antarctic. Antarctic ecology, vol. 1. London: Academic Press, 61-162.

Scott J.J. \& J.B. Kirkpatrick. 2008. Rabbits, landslips and vegetation change on the coastal slopes of subantarctic Macquarie Island, 1980-2007: implications for management. Polar Biology, 31: 409-419.

Volonterio O., De Leon R.P., Convey P. \& E. Krzeminska. 2013. First record of Trichoceridae (Diptera) in the maritime Antarctic. Polar Biology, 36: 1125-1131 DOI 10.1007/s00300-013-13344.

Whinam J., Chilcott N. \& D.M. Bergstrom. 2005. Subantarctic hitchhikers: expeditionary as vectors for the introduction of alien organisms. Biological Conservation, 121: 207-219.

Fecha de Recepción: 14 de septiembre de 2020 Fecha de Aceptación: 02 de diciembre 2020 